\title{
Generation of the Stream of Consciousness by Neuronal Synaptic Interaction
}

\author{
Andrew Duggins 1,2* \\ 1 Western Clinical School, Faculty of Medicine and Health, University of Sydney; \\ andrew.duggins@sydney.edu.au \\ 2 Department of Neurology, Westmead Hospital; andrew.duggins@health.nsw.gov.au \\ * Correspondence: andrew.duggins@sydney.edu.au; Tel.: +61-413-475-681
}

\begin{abstract}
In previous work, a quantum mathematical formalism associated an element of experience with a single sensory neuron, as a local reduction of a global mental state. In contrast to the binary objective states of neuronal polarisation/depolarisation, neuronal experience was modeled as a continuous variable, the instantaneous value of which could only be estimated statistically from an ensemble of evoked responses to stereotyped stimulus presentation. In the present work, the quantum operations formalism of energy dissipation through amplitude damping is adopted to explain how smooth evolution of conscious experience might arise from discrete spikes and discontinuous synaptic transmission between neurons.
\end{abstract}

Keywords: stream of consciousness; quantum mechanics; decoherence theory; synaptic transmission; spike; action potential; neural code; neural correlate of consciousness

\section{Introduction}

\subsection{Conceptual Background}

At the click of a Geiger counter, an observer infers that a nearby Americium-74 nucleus has decayed to Neptunium-72, emitting an alpha particle. Yet before and after this nuclear transmutation, the universe is in a coherent superposition of states of parent and of daughter radionuclide. Moreover, the state vector of the universe moves smoothly and continuously through Hilbert space over the lifetime of the radionuclide from the parent to daughter eigenspace, a decay that had appeared spontaneous and random generated by the time-independent interaction between nucleus and its immediate environment. Even considering only the reduced state of this one nucleus, the probability weighting of the parent in an incoherent mixture with daughter radionuclide must smoothly decay over time. These dynamics only appear as an abrupt transition because out of the mixture of parent and daughter radionuclides only one or other can be manifest in the world of the observer at a point in time.

This account raises the question whether other apparently discrete spontaneous events arising in the natural world might be generated similarly. This would be particularly plausible where transition events in subsystems were known to correlate with the state and smooth trajectory of a spatially distributed system of which subsystems were all part. The brain is such a multipartite and anatomically distributed system, in which the stream of unified consciousness is driven by 
transition events in neuronal components. Specifically, we consider whether a single spike, and the chemical synaptic transmission that results, might be modelled as a continuous interaction between an efferent and afferent neuron.

\subsection{Existing Model: axioms and quantum foundations}

In the author's previous work, two 'bridging principles' for the mind-brain relation were presented as axiomatic foundations of a neuronal model of sensory perception. The first postulate was that the "contribution of a single sensory neuron to the neural code for the sensory environment parallels the relationship between a neuronal element of experience and the overall sensory percept" [1]. As an example, if as suggested by the experiment of Britten [2] the organism's behavioural response to direction of visual motion, within a limited region of the visual field, can be predicted almost entirely by the response of a single, carefully selected neuron in the motionsensitive cortex V5, then the neuron singlehandedly encodes this aspect of the sensory environment. The first bridging principle goes further to say that such a V5 neuron also affords, within a limited region, this same limited aspect of motion experience. The second postulate extended the mindbrain parallel to neuronal relations: "however the similarity between environmental features specified by a pair of neurons is encoded in their joint activity, this objective association parallels the qualitative proximity of respective neuronal elements of experience" [3]. Previous work emphasised perceptual rather than conceptual experience, but in what follows it will be assumed that these bridging principles can be extended to a more general correspondence between objective and subjective representation of information. A third and final bridging principle was suggested in section 1.1 of the current paper, but is here stated again formally: neuronal synaptic transmission generates not only the evolution of an objective neural representation of information but also the dynamics of conscious experience (those elements of experience and their relations corresponding to that objective representation by the first and second bridging principles).

Notwithstanding the close association, implied by these bridging principles, between elements of experience and their neural correlates, a purely physicalist approach, it was argued, would fail to capture two essential features of consciousness, subjectivity and unity. A starting point to the development of a model to describe these features was the principle of evoked responses in neurophysiology. Neural responses are quantised as spikes. Over an ensemble of stereotyped stimulus presentations, the best description of a sensory neuron's response to the stimulus at a point in time is its instantaneous firing rate, averaged across trials, not the number of spikes that have already occurred on an individual trial, or the objective state of the neuron that pertains at that moment. The quantum mathematical formalism is consistent with an interpretation that the state of a system really exists and has statistical effects over an ensemble of trials with identical starting conditions, even though the state itself is not directly measurable. In the quantum mechanical mathematical formalism of neural signaling that was developed, this feature of the quantum state was identified with the subjectivity of a neural element of experience. Spikes were considered to be objective transition events between definite levels of neuronal potential, even as an element of experience, inaccessible to direct measurement, smoothly decayed (like the quantum conception of alpha decay mentioned above). When the model was applied to the neurophysiologic scenario of repeated stereotyped stimulus presentations, the expectation value of spike potential across trials 
was not only proportional to the instantaneous firing rate, but also to a scalar quantity of experience inherent in the inaccessible neural state (a 'perception value', see below).

If only this part of the quantum formalism had been appropriated, consciousness would have been neuro-anatomically locally explicable, consistent with the 'microconsciousness' theory of Zeki: "Activity in each separate processing node generates a microconsciousness for the attribute for which that node is specialised. Consequently, there are several microconsciousnesses, corresponding to the activity of cells at different nodes within different processing systems" [4]. It seemed to the author that such "summative atomism" was a poor description of the phenomenon: "a sub-mind is an atrocious monstrosity, just as is a plural-mind - neither having any counterpart in anybody's experience, neither being in any way imaginable" [5]. Indeed, the unity of consciousness seemed to be a fundamental feature. A unified consciousness was considered to be singular and not plural; complete in itself, not a fraction of a greater whole. In contrast, sensation, cognition, emotion and volition were considered not complete experiences but aspects of the subject's consciousness. Whatever level of perceptual or conceptual experience is introspected upon, it always seemed possible to consider a higher level at which the relation between simultaneous percepts or concepts is experienced. The unified mental state was thus proposed to exist at the apex of a hierarchy of bound percepts and concepts. The author shared with Nagel the view that "It seems inevitable that psychophysical explanation will apply first at the level of some kind of elements of experience; but if these elements come together in a single consciousness, they must also be components of a single point of view" [6]. This suggested that, just as the bound percept might be resolved along multiple perceptual dimensions, perhaps a unified consciousness might usefully be represented as a mental state vector in a subjective space spanned by all of the dimensions of perceptual experience.

With these ideas in mind, two other features of the quantum mathematical formalism, superposition of states and tensor product combination of state spaces, were applied to a neuronal model that had been extended to the whole brain. This facilitated description of the mental state as a pure, inseparable state (a vector or uni-dimensional projector) on this combined system of vast dimension. There was proposed a direct correspondence between the unity of experience, a property of the system as a whole, and such coherence of the state of that system. The reduced state of any subsystem of one or many neurons would be a mixed-state density operator. Were this not so, the completeness privilege of consciousness described above would be lost. Such a mental state would be not only unified but also emergent: it would reduce to, but be not entirely constituted in the semi-classical phenomenal correlates of firing rate at the single neuron level, mentioned above. The objective manifestation of an inseparable pure mental state would be firing correlations between neurons in anatomically remote regions that have no direct synaptic connection with each other, perhaps correlations that could not have been established classically by synaptic input from a common source.

In a decoherence theoretic interpretation of quantum mechanics, the emergence of a probability-weighted mixture of discrete objective states is the local manifestation of a smooth, unitary evolution generated by the interaction between a system and its environment [7]. In the further development of the neuronal model of consciousness to be presented below, it is proposed 
that such a decoherence theoretic framework can reconcile discontinuous synaptic transmission with the stream of consciousness. Specifically, complex neuronal vector spaces, which might have seemed an unnecessary extravagance when modelling subjectivity and unity, will facilitate a description of unitary evolution of the mental state ${ }^{1}$. Of course, if this were merely the application of quantum physics to the brain, considered as an open quantum system, its quantum state would no longer remain pure after a vanishingly small decoherence time, although the state of a very large composite system including the brain and some part of its physical environment would remain pure. In order to maintain the picture of a pure mental state, the only interaction that will be considered in this neuronal model is that occurring at the synapse. Interactions that occur between sub-neural structures and their physical environment will be neglected.

Of course, classical neural dynamics, generated by synaptic transmission, are already well described. At a synapse on the dendrite of a recipient neuron, excitatory neuro-transmitters, released as a spike depolarises the pre-synaptic terminal, increase sodium permeability by opening chemically-gated channels and partially depolarise the post-synaptic membrane. Partial depolarisation at the trigger zone, by passive (or 'electrotonic') conduction of this excitatory postsynaptic potential, may reach a threshold at which voltage-gated sodium channels open, making the transmembrane potential abruptly positive. This opens similar channels in the adjacent neural membrane, so that complete depolarisation extends rapidly throughout the neuron as a spike. What is to be presented here will challenge none of this. Partly this is because the model is a coarsegrained description, neglecting all this subcellular activity to represent a spike as nothing more than a Dirac delta function. More importantly though, the contention is that the continuous evolution of the mental state reduces to the discrete synaptic transmission of information of the classical neuron doctrine at a local level of explanation, in the same way that the click of the Geiger counter is a local manifestation of the unitary evolution of the state vector of the universe.

\subsection{Existing Model: mathematical formalism}

In previous work, a unified consciousness, or mental state, was modelled as a vector on a composite tensor product of single neuronal complex vector spaces [3]. Each of these neuronal spaces was spanned by an orthonormal basis of integer 'action potential' states $|n\rangle$. These were eigenvectors of a hermitian number operator $N$ : states of inevitability of corresponding integer spike counts $n$, but in contradistinction to conventional neuroscience not the depolarisation events themselves. A superposition principle was introduced in which pure states $|\psi\rangle$, sums of integer action potential states weighted by complex amplitudes $\langle n \mid \psi\rangle$, were all valid single neuronal states. If a state $|\psi\rangle$ were normalised, the squared modulus $|\langle n \mid \psi\rangle|^{2}$ would be the prior probability that a neuron in that state would ultimately spike $n$ times, and the expectation value of $N,\langle N\rangle$, a sum of eigenvalues $n$ weighted by these respective probabilities, would be the expected spike count. The proposed equivalence of using the uni-dimensional projector $|\psi\rangle\langle\psi|$ or the vector $|\psi\rangle$ for the description of a pure state of a neuron implied that a global phase $e^{i \theta}$ across amplitudes $\langle n \mid \psi\rangle$

\footnotetext{
${ }^{1}$ Unitary evolution implies that the evolution operator when multiplied by its adjoint yields the identity (see section 3.1 Unitary dynamics of isolated neurons). It is a concept unrelated to the unity of consciousness, modelled in this formulation as an inseparable, pure mental state of the composite neuronal system.
} 
would have no subjective or objective meaning. The significance of relative phase differences (which remain in either description) will be revealed below.

Application of the same superposition principle to the composite space yielded entangled mental states that were not tensor products of single neuronal states, putting the concept of emergence of consciousness in mathematical form [3]. Consciousness still had a neural correlate, however, in that local reduction of the pure mental state to the single neuron $A$ was shown to be the density matrix $\rho^{A}$, a probability-weighted mixture of uni-dimensional projectors. A 'memoryless firing' assumption, in which the posterior probability of a future spike would be unaffected by a period of neuronal silence, implied that the instantaneous firing rate would be proportional to the expected spike count with proportionality constant $w$ [1]. In the same way that the instantaneous firing rate might quantify a single sensory neuron's contribution to the objective neural code for an environmental stimulus, it was proposed that a scalar 'perception value', also proportional to $\langle N\rangle$ but with constant $r$, would quantify that same neuron's contribution to the subjective percept. Similarly, instantaneous firing rate dependence, a manifestation within a neuronal pair of an entangled mental state, might quantify both an objective inter-neuronal code and subjective phenomenal proximity between neural elements of experience [3].

\section{Materials and Methods}

Here we argue reductio ad absurdum that without synaptic transmission, action potentials would remain sequestered in neurons, perception values would be unchanging and consciousness static. We extend the quantum mechanical formalism summarised above to show that a single neuron isolated in this way could nevertheless undergo a unitary evolution generated by $N$ in which integer action potential eigenstates remain unchanged but for acquisition of a relative phase $-n \omega t$. We extrapolate that in any neural system in which, by virtue of isolation, there is conservation of action potential, the subjective state of that system could nevertheless undergo a unitary evolution $U$. But synaptic interaction within that system would require that the eigenvectors of $U$ were no longer single neuron integer action potential eigenstates. Applying this principle to a hypothetical isolated 2-neuron system, we model the oscillatory flow of a single action potential between component neurons $A$ and $B$ by synaptic transmission as a Jaynes-Cummings interaction [8]. We use an operator sum formalism to reduce this unitary evolution to a description of the continuous evolution of the single neuron state $\rho^{B}$. We qualify the perception value concept, replacing the general constant $r$ with 'nervous energy': a neuron-specific experience-per-spike dependent on $\omega$. We show how, in a realistic brain of polysynaptic neuronal interaction, this allows reconciliation of oscillatory flow of action potential between a neuronal pair with exponential decay in firing of the efferent neuron, of rate constant $w$, predicted by the memoryless firing assumption [1]. 


\section{Results}

\subsection{Unitary dynamics of isolated neurons}

If we were able to set $w=0$, and in doing so abruptly impose upon the brain complete neuronal isolation, then a neuron's action potential could no longer be realised as spikes. If a single neuron were in a pure state $|\psi\rangle$ with probability $|\langle n \mid \psi\rangle|^{2}$ of $n$ eventual spikes prior to this imposed isolation, it would still have the same probability of $n$ spikes after such isolation were relieved. While synaptic transmission to or from the neuron remained impossible, $\langle N\rangle$ and perception value would remain constant. In the proposed mathematical formalism the evolution of single neuronal elements of experience is entirely attributable to spikes and synaptic transmission.

Neuronal isolation would nevertheless be compatible with an evolution in which, over time $t$, incremental integer action potential states acquire a relative phase $\omega t$. This implies an association between relative phase and experience that will be justified below. An operator $U$ on the single neuron space to effect such an evolution would be unitary, meaning that $U^{\dagger} U$ is the identity $I$ for example the operator

$$
\begin{aligned}
& U(t)=\sum_{n} e^{-i n \omega t}|n\rangle\langle n| \\
& U^{\dagger}(t) U(t)=\sum_{n}|n\rangle\langle n|
\end{aligned}
$$

This particular $U$ is generated by $N$, and leaves the phase of the ground state $|0\rangle$ unchanged. Of course any operator that imposed the same relative phase but a different global phase on the neural state would describe an identical evolution. The evolution of an isolated single neuron in a superposition of 0 - and 1-action potentials is depicted in Figure 1. It will be mathematically convenient, when we consider the contribution of such single-neuron evolution to a multi-neuron system, to use an equivalent $U$, generated by an operator sharing with $N$ eigenvectors belonging to eigenvalues of unit separation (so that they again acquire relative phase $-\omega t$ ), presented here in matrix form with respect to the basis $\{|n\rangle\}$ :

$$
-\frac{Z}{2}=\left[\begin{array}{cc}
-\frac{1}{2} & 0 \\
0 & \frac{1}{2}
\end{array}\right]
$$

In a 2-neuron system within which individual neurons were isolated, an evolution on the composite space could only impose a relative phase on tensor products of single neuron integer action potential states, and could neither introduce nor abolish entanglement in a pure state superposition thereof. The quality of a very limited consciousness would remain static. The tensor product of single neuron unitary operators to effect this evolution, $U^{A} \otimes U^{B}$ might be generated by an 'uncoupled' operator [4]

$$
-\frac{Z_{A}}{2} \otimes I^{B}+I^{A} \otimes-\frac{Z_{B}}{2}=\left[\begin{array}{cccc}
-1 & 0 & 0 & 0 \\
0 & 0 & 0 & 0 \\
0 & 0 & 0 & 0 \\
0 & 0 & 0 & 1
\end{array}\right]
$$

where the choice of $-\frac{Z}{2}$ leaves the phase of the 1-action potential eigenspace unchanged. 


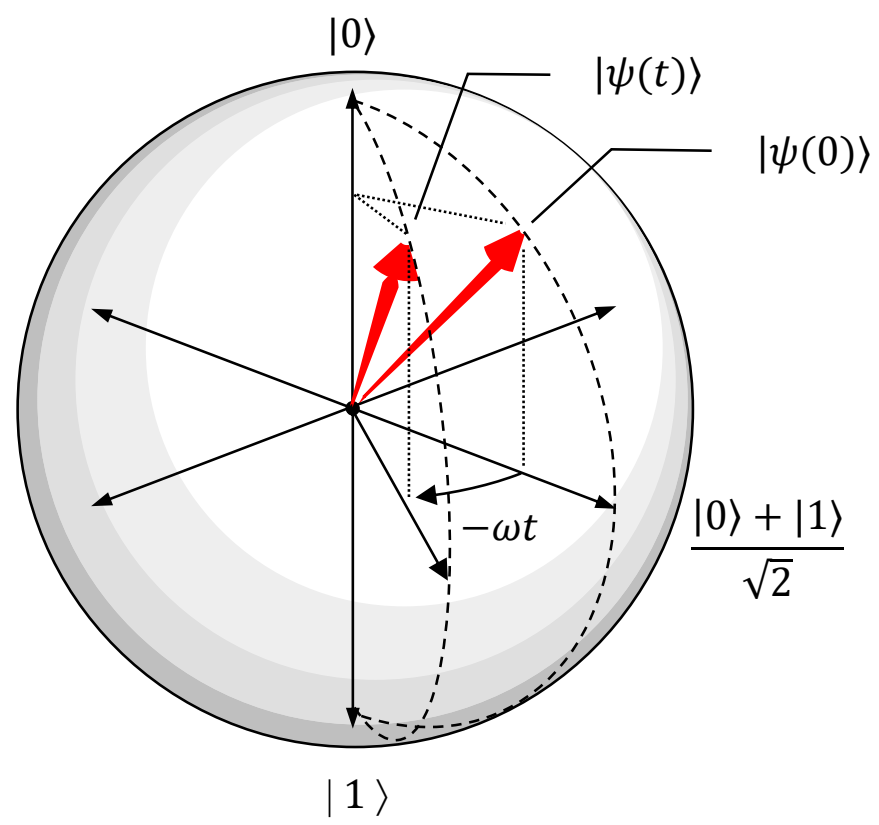

Figure 1. Evolution on the Bloch sphere of a superposition state of an isolated single neuron: $|\psi(0)\rangle=\langle 0 \mid \psi(0)\rangle|0\rangle+\langle 1 \mid \psi(0)\rangle|1\rangle$. $|\psi(0)\rangle$ is assumed to have a relative phase of 0 . The one-action potential state acquires a phase of $-\omega t$ over time $t$ ( $\omega$ assumed positive, phase acquisition negative), relative to the zero-action potential state.

\subsection{Unitary dynamics of synaptic interaction}

More generally of course, a multi-neuron system might be isolated, in the sense that there could be no synaptic transmission to or from the wider neural environment, even as such interaction was ongoing between component neurons. Action potential would be conserved within the system, even as it flowed between components. Within a 2-neuron system (Figure 2), an interaction between neurons might generate a unitary evolution that would not preserve $\left|0^{A}\right\rangle\left|1^{B}\right\rangle$ or $\left|1^{A}\right\rangle\left|0^{B}\right\rangle$ eigenvectors, but would nevertheless conserve one action potential.

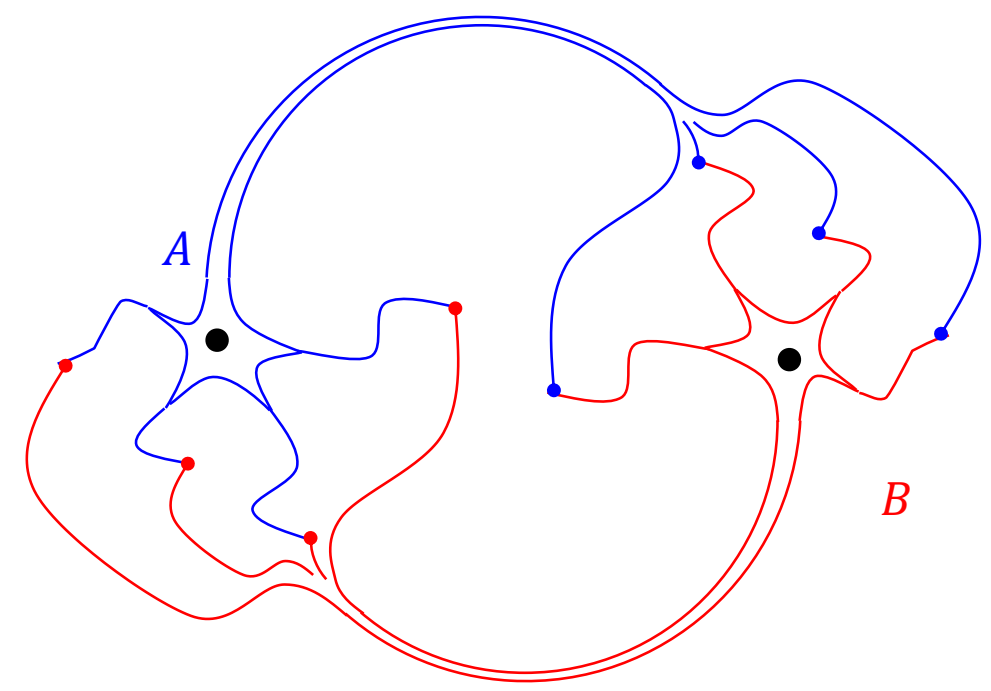

Figure 2. A fictitious isolated 2-neuron system. Like the isolated single neuron, the 2-neuron system should undergo unitary evolution, without gain or loss of action potential. Here though, potential should flow between neurons as spikes. 
If neuron $B$ is initially in the 1-action potential state, while $A$ is initially in the ground state, then with prior probability $P(t)$, after some brief time $t$ has elapsed, a single spike may have occurred in $B$. The reduced state of neuron $B$ will then be in a mixture of the 0 -action potential state weighted by probability $P(t)$ and the 1-action potential state weighted by the probability $1-P(t)$ :

$$
\rho^{B}=P(t)\left|0^{B}\right\rangle\left\langle 0^{B}|+(1-P(t))| 1^{B}\right\rangle\left\langle 1^{B}\right|
$$

This is consistent with an evolution that takes the pure initial state $\left|0^{A}\right\rangle\left|1^{B}\right\rangle$ to a weighted superposition of $\left|0^{A}\right\rangle\left|1^{B}\right\rangle$ and $\left|1^{A}\right\rangle\left|0^{B}\right\rangle$, where the squared modulus of the amplitude of the second term is the firing probability:

$$
\begin{gathered}
\left|0^{A}\right\rangle\left|1^{B}\right\rangle \stackrel{t}{\rightarrow} \sqrt{1-P(t)}\left|0^{A}\right\rangle\left|1^{B}\right\rangle-i \sqrt{P(t)}\left|1^{A}\right\rangle\left|0^{B}\right\rangle \\
{\left[\begin{array}{l}
0 \\
1 \\
0 \\
0
\end{array}\right] \stackrel{t}{\rightarrow}\left[\begin{array}{c}
0 \\
\sqrt{1-P(t)} \\
-i \sqrt{P(t)} \\
0
\end{array}\right]}
\end{gathered}
$$

As in the non-interacting neuron model above, we choose an evolution in which $\left|0^{A}\right\rangle\left|0^{B}\right\rangle$ and $\left|1^{A}\right\rangle\left|1^{B}\right\rangle$ acquire a positive and negative phase respectively, determined by single neuron evolution, but the phase of the $\left|0^{A}\right\rangle\left|1^{B}\right\rangle$ term in the superposition does not change. The phase of the $\left|1^{A}\right\rangle\left|0^{B}\right\rangle$ term is chosen to simplify the mathematical form of the operator that generates this evolution.

The columns of the operator that achieves these transformations, $U^{A B}(t)$, are orthonormal (since $U^{A B}$ is unitary) and reflect the symmetry of the system:

$$
U^{A B}(t)=\left[\begin{array}{cccc}
e^{i \omega t} & 0 & 0 & 0 \\
0 & \sqrt{1-P(t)} & -i \sqrt{P(t)} & 0 \\
0 & -i \sqrt{P(t)} & \sqrt{1-P(t)} & 0 \\
0 & 0 & 0 & e^{-i \omega t}
\end{array}\right]
$$

\subsection{Evolution of the reduced neural state: spike elements}

More generally still, if neuron $B$ is initially in a superposition of 0 - and 1-action potential states weighted by $c_{0}$ and $c_{1}$ respectively (and neuron $A$ is again assumed to start in the ground state) then a matrix equation for the unitary evolution of the initial product state is

$$
\begin{gathered}
{\left[\begin{array}{cccc}
e^{i \omega t} & 0 & 0 & 0 \\
0 & \sqrt{1-P(t)} & -i \sqrt{P(t)} & 0 \\
0 & -i \sqrt{P(t)} & \sqrt{1-P(t)} & 0 \\
0 & 0 & 0 & e^{-i \omega t}
\end{array}\right] \times\left[\begin{array}{c}
c_{0} \\
c_{1} \\
0 \\
0
\end{array}\right]=\left[\begin{array}{c}
c_{0} e^{i \omega t} \\
c_{1} \sqrt{1-P(t)} \\
0 \\
0
\end{array}\right]+\left[\begin{array}{c}
0 \\
0 \\
-c_{1} i \sqrt{P(t)} \\
0
\end{array}\right]} \\
U^{A B}(t)\left|0^{A}\right\rangle \otimes\left|\psi^{B}(0)\right\rangle=\left|0^{A}\right\rangle \otimes S_{0}(t)\left|\psi^{B}(0)\right\rangle+\left|1^{A}\right\rangle \otimes S_{1}(t)\left|\psi^{B}(0)\right\rangle
\end{gathered}
$$

where 'spike elements' $S_{0}$ and $S_{1}$ are operators $\left\langle 0^{A}\left|U^{A B}\right| 0^{A}\right\rangle$ and $\left\langle 1^{A}\left|U^{A B}\right| 0^{A}\right\rangle$ on $B$, representations of which are the submatrices of $U^{A B},\left[\begin{array}{cc}e^{i \omega t} & 0 \\ 0 & \sqrt{1-P(t)}\end{array}\right]$ and $\left[\begin{array}{cc}0 & -i \sqrt{ }(P(t) \\ 0 & 0\end{array}\right]$ respectively. The spike probability is the squared modulus of the second term

$$
\left\langle\psi^{B}(0)\left|S_{1}(t)^{\dagger} S_{1}(t)\right| \psi^{B}(0)\right\rangle=P(t)\left|c_{1}\right|^{2}
$$

Spike elements decompose the transformation of $\rho^{B}$ through synaptic transmission: 


$$
\left|\psi^{B}(0)\right\rangle\left\langle\psi^{B}(0)\left|\rightarrow S_{0}\right| \psi^{B}(0)\right\rangle\left\langle\psi^{B}(0)\left|S_{0}^{\dagger}+S_{1}\right| \psi^{B}(0)\right\rangle\left\langle\psi^{B}(0)\right| S_{1}^{\dagger}
$$

as illustrated in Figure 3, and in matrix form:

$$
\left[\begin{array}{cc}
\left|c_{0}\right|^{2} & c_{0} c_{1}{ }^{*} \\
c_{1} c_{0}{ }^{*} & \left|c_{1}\right|^{2}
\end{array}\right] \stackrel{t}{\rightarrow}\left[\begin{array}{cc}
e^{2 i \omega t}\left|c_{0}\right|^{2}+P(t)\left|c_{1}\right|^{2} & e^{i \omega t} \sqrt{1-P(t)} c_{0} c_{1}{ }^{*} \\
e^{i \omega t} \sqrt{1-P(t)} c_{1} c_{0}{ }^{*} & (1-P(t))\left|c_{1}\right|^{2}
\end{array}\right]
$$

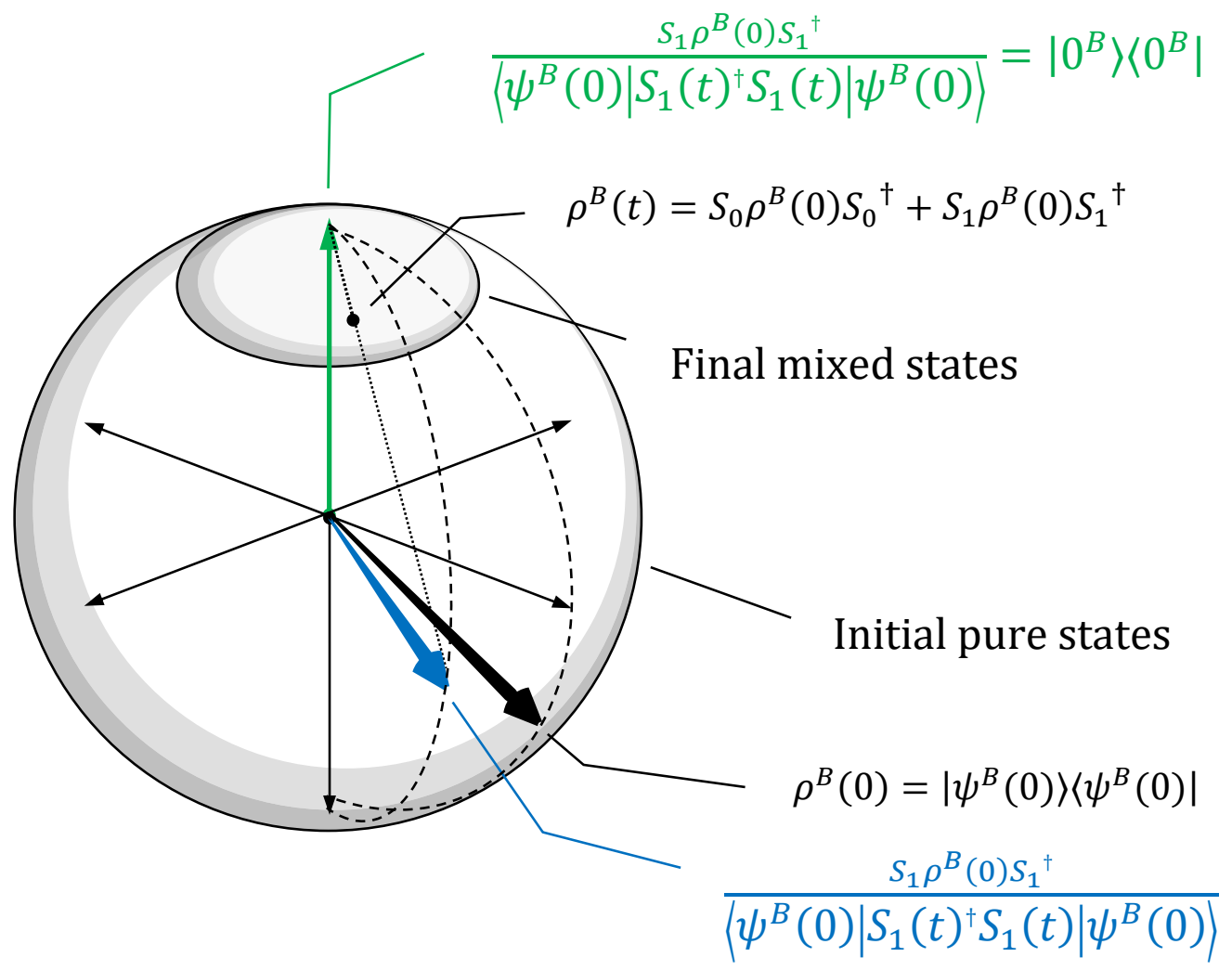

Figure 3. Evolution of the reduced state of neuron $B$, part of an isolated 2-neuron system as in Figure 2. Here the initial composite state $\left|0^{A}\right\rangle\left|\psi^{B}(0)\right\rangle$ is separable, so the initial reduced state $\rho^{B}(0)$ is pure, depicted as a black arrow on the surface of the Bloch sphere. Unitary evolution of the combined system is equivalent to the operation of spike elements $S_{0}$ and $S_{1}$ on the neural space of $B$. After a brief period of time, in which the probability of a spike in neuron $B$ is approximately $\left\langle\psi^{B}(0)\left|S_{1}(t)^{\dagger} S_{1}(t)\right| \psi^{B}(0)\right\rangle$, the reduced state evolves smoothly to a weighted mixture $\rho^{B}(t)$ of the zero-action potential state (green arrow) and the no-spike pure state (blue arrow), that mixture depicted as a point in the interior of the sphere. 


\subsection{Synaptic Interaction on the 1-action potential manifold: Part I}

Entangled states of 1 -action potential $\frac{1}{\sqrt{2}}\left(\left|0^{A}\right\rangle\left|1^{B}\right\rangle \pm\left|1^{A}\right\rangle\left|0^{B}\right\rangle\right)$ are unchanged by the operation of $U^{A B}$, but for the acquisition of relative phase. On the 1-action potential manifold of states, these states are also eigenvectors of an interaction operator $X$. We might insist, as previously, that relative phase must accumulate linearly over time, in which case $X$ generates an evolution on this manifold

$$
e^{-i X g t}
$$

where $g$ parameterises the strength of synaptic coupling (Figure 4). We can equate this with the central submatrix of $U^{A B}(t)$, substituting $\sin ^{2} g t$ for the prior probability of a single spike $P(t)$ :

$$
\left[\begin{array}{cc}
\cos g t & -i \sin g t \\
-i \sin g t & \cos g t
\end{array}\right]
$$

The action potential then 'sloshes' back and forth between neurons at a frequency of $\frac{g}{\pi}$.

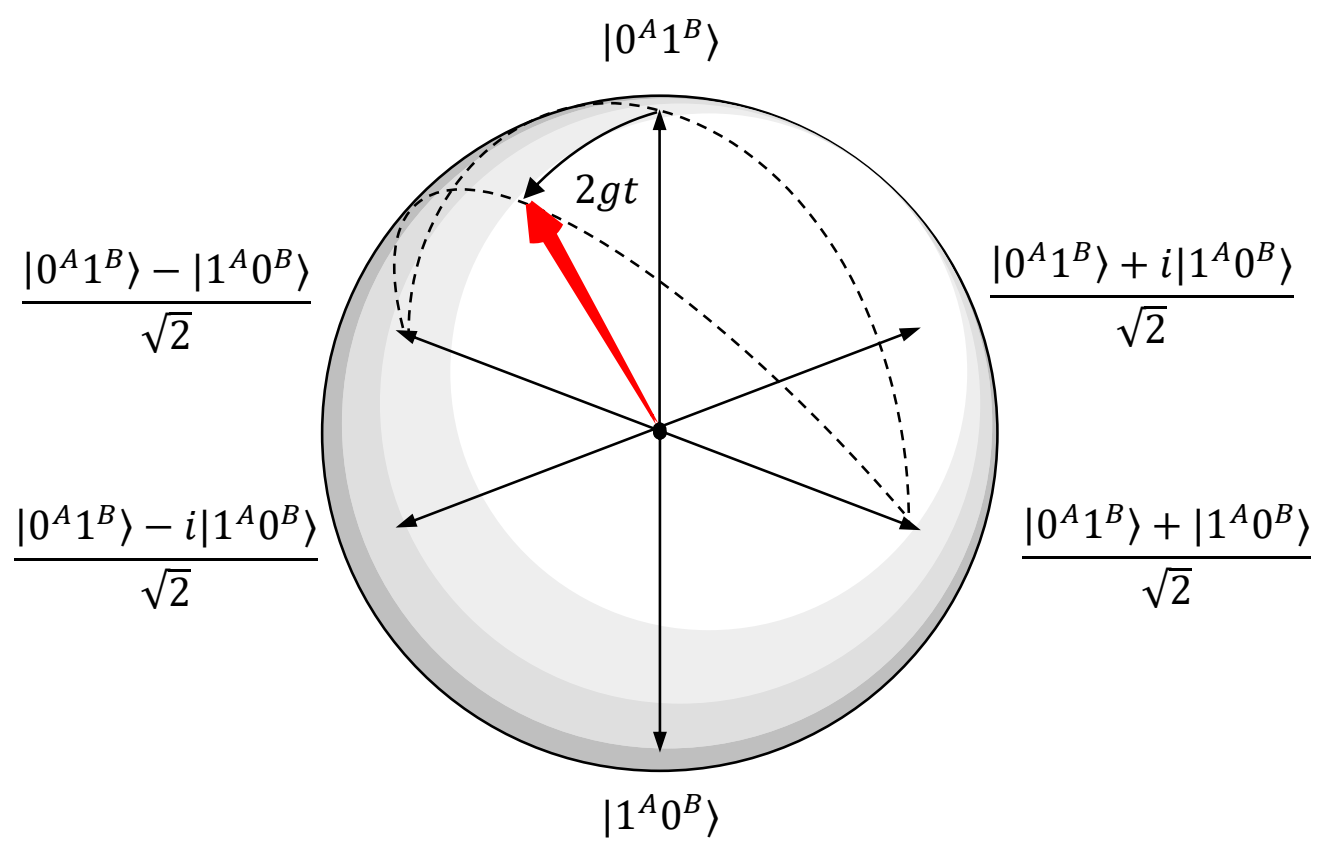

Figure 4. The $\left|0^{A} 1^{B}\right\rangle,\left|1^{A} 0^{B}\right\rangle$ manifold of states of the isolated 2-neuron system in Figure 2, depicted as a Bloch sphere. After a brief period of time $t$, the initial separable state $\left|0^{A} 1^{B}\right\rangle$ has evolved to the entangled state $\cos g t\left|0^{A} 1^{B}\right\rangle-i \sin g t\left|1^{A} 0^{B}\right\rangle$, reflecting a spike probability in neuron $B$ of approximately $\sin ^{2} g t$. 


\subsection{A paradox: oscillation versus decay}

There is an inconsistency here with our earlier prediction that $\langle N\rangle$ and perception value should be proportional to a neuron's instantaneous firing rate (Figure 5a). An instantaneous firing rate must be the gradient with which spike probability increases over time. But in the unitary evolution that we have described, over a brief time period $t$

$$
P(t)=\sin ^{2} g t \cong g^{2} t^{2}
$$

so the instantaneous firing rate of neuron $B$ increases in proportion to time:

$$
f_{B}(t) \cong 2 g^{2} t
$$

Initially, when $\left\langle N^{B}\right\rangle$ is maximal, the instantaneous firing rate is zero (Figure $5 b$ )!

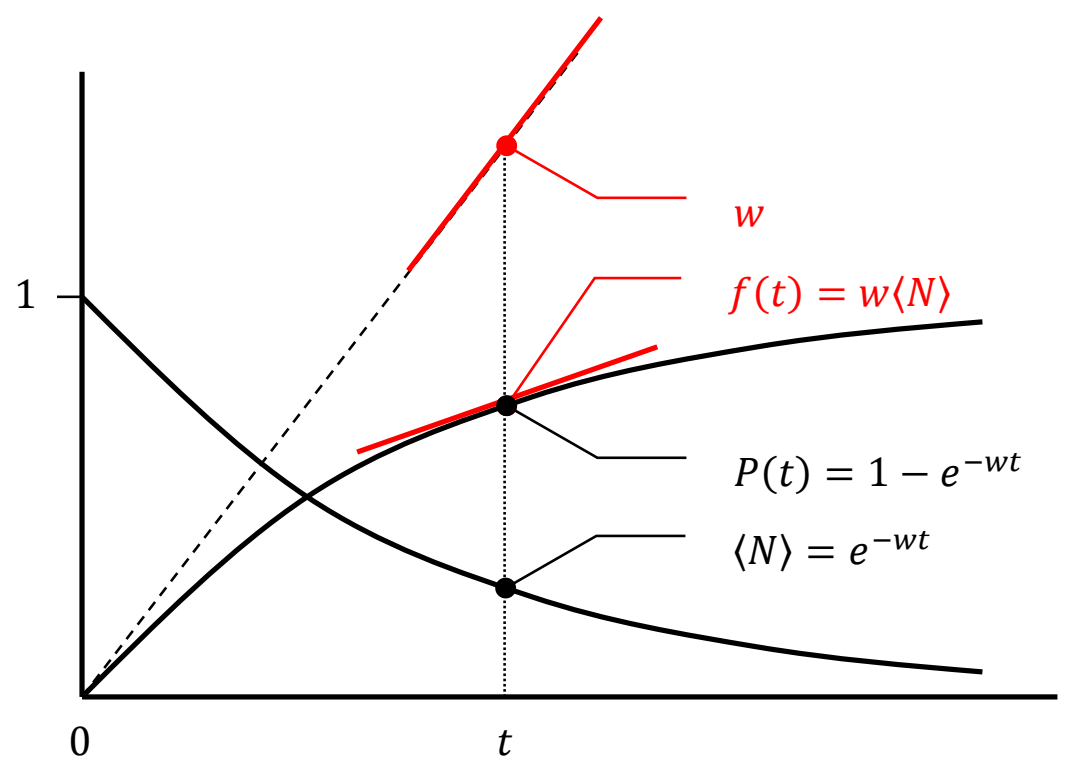

Figure 5a. Changes in $\langle N\rangle$; spike probability $P(t)$; and instantaneous firing rate $f(t)$ in an interacting neuron initially in the one-action potential state, according to the 'memoryless firing' assumption. Compared with Figure $5 b$, the instantaneous firing rate decays exponentially over time from an initial rate constant of $w$, remaining proportional to $\langle N\rangle$. 
48

49

50

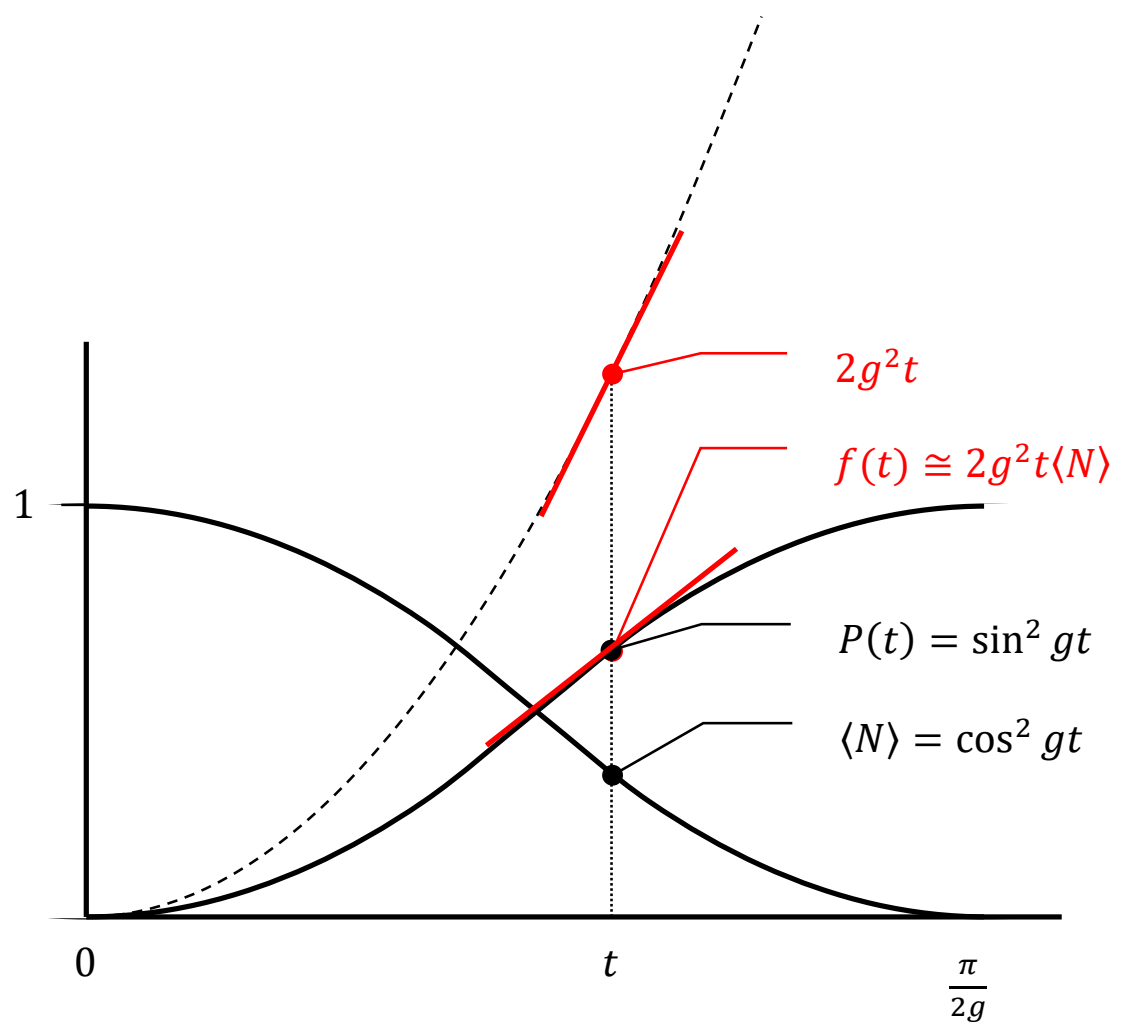

Figure 5b. Changes in $\langle N\rangle$; spike probability $P(t)$; and rate of accumulation of spike probability, the instantaneous firing rate $f(t)$ in neuron $B$, part of the isolated 2-neuron system in Figure 2 that is initially in the state $\left|0^{A} 1^{B}\right\rangle$. Initially, instantaneous firing rate increases with time. These changes correspond to the evolution of the composite state depicted in Figure 4, but they do not accord with the prediction that instantaneous firing rate should vary with $\langle N\rangle$.

The perception value was supposed to be a general description of the subjective correlate of the neural response on a single trial, compatible with the instantaneous firing rate as a description of the objective neural response over an infinite ensemble of stimulus repetitions. We would be loath to abandon the simple linear relationship between perception value and firing rate that follows from the memoryless firing assumption [1]. Yet the current model is particularly attractive in that it links the dynamics of experience to synaptic interaction, as most neuroscientists would require. In fact, reconciliation between these positions is possible, if we recognise and overcome a limitation of the current model.

\subsection{Variable nervous energy of the action potential}

We must reject the implicit assumption that different neurons with the same spike potential (the same expected spike count $\langle N\rangle$ ) should afford the same perception value (we cannot assume that the proportionality constant $r$, between $\langle N\rangle$ and the perception value, is the same for different neurons). While it would seem essential, if a quantum formalism is to have any validity in modeling brain activity, for all action potentials in a particular neuron to be identical, action potentials of a pre-synaptic efferent and post-synaptic 
74

afferent neuron could differ. We have not uncovered any objective manifestation of the value of $\omega$, so it would seem reasonable to allow the level of perceptual experience that could be attributed to a neuron at a given instantaneous firing rate to be determined by a neuron-specific $\omega$, if that would lead to a resolution of the paradox.

To achieve this, we will need a 'nervous energy' operator on the neuronal space

$$
H=\hbar \omega N
$$

where $\hbar$ is a fundamental constant across all neurons, and $\hbar \omega$ replaces $r$. The nervous energy of 1 action potential

$$
\langle 1|H| 1\rangle=\hbar \omega
$$

is the 'experience-per-spike' of that neuron. Perception is an immeasurable quantity, and a neuron's contribution to consciousness can only be defined relative to other neurons, so we might reasonably allocate $\hbar$ a value of 1 .

\subsection{Synaptic Interaction on the 1-action potential manifold: Part II}

The isolated single neuron will now evolve as

$$
U(t)=e^{-i H t}
$$

The uncoupled operator that generates the evolution of the 2-neuron system of isolated individual neurons becomes:

$$
-\omega^{A} \frac{Z^{A}}{2} \otimes I^{B}-I^{A} \otimes \omega^{B} \frac{Z^{B}}{2}
$$

This adds a contribution $\delta$ to the nervous energy of synaptic interaction on the one action potential manifold

$$
\left[\begin{array}{cc}
\delta & g \\
g & -\delta
\end{array}\right]
$$

where $\delta$ is the deviation of each $\omega$ from the average

$$
\delta=\frac{\left(\omega^{B}-\omega^{A}\right)}{2}
$$

If we define a Rabi frequency

$$
\Omega=\sqrt{g^{2}-\delta^{2}}
$$

then the evolution generated on this manifold, illustrated in Figure 6, becomes

$$
\left[\begin{array}{cc}
\cos \Omega t-i\left(\frac{\delta}{\Omega}\right) \sin \Omega t & -i\left(\frac{g}{\Omega}\right) \sin \Omega t \\
-i\left(\frac{g}{\Omega}\right) \sin \Omega t & \cos \Omega t+i\left(\frac{\delta}{\Omega}\right) \sin \Omega t
\end{array}\right]
$$

There is still an oscillatory flow of action potential, but as the discrepancy in $\omega$ between efferent and afferent neuron increases, the weighting of the 1 -action potential state in $\rho^{B}(t)$ dips to a lesser extent $\left(\frac{g^{2}}{g^{2}+\delta^{2}}\right)$ and is more promptly restored (period $\frac{\pi}{\Omega}$ ). 


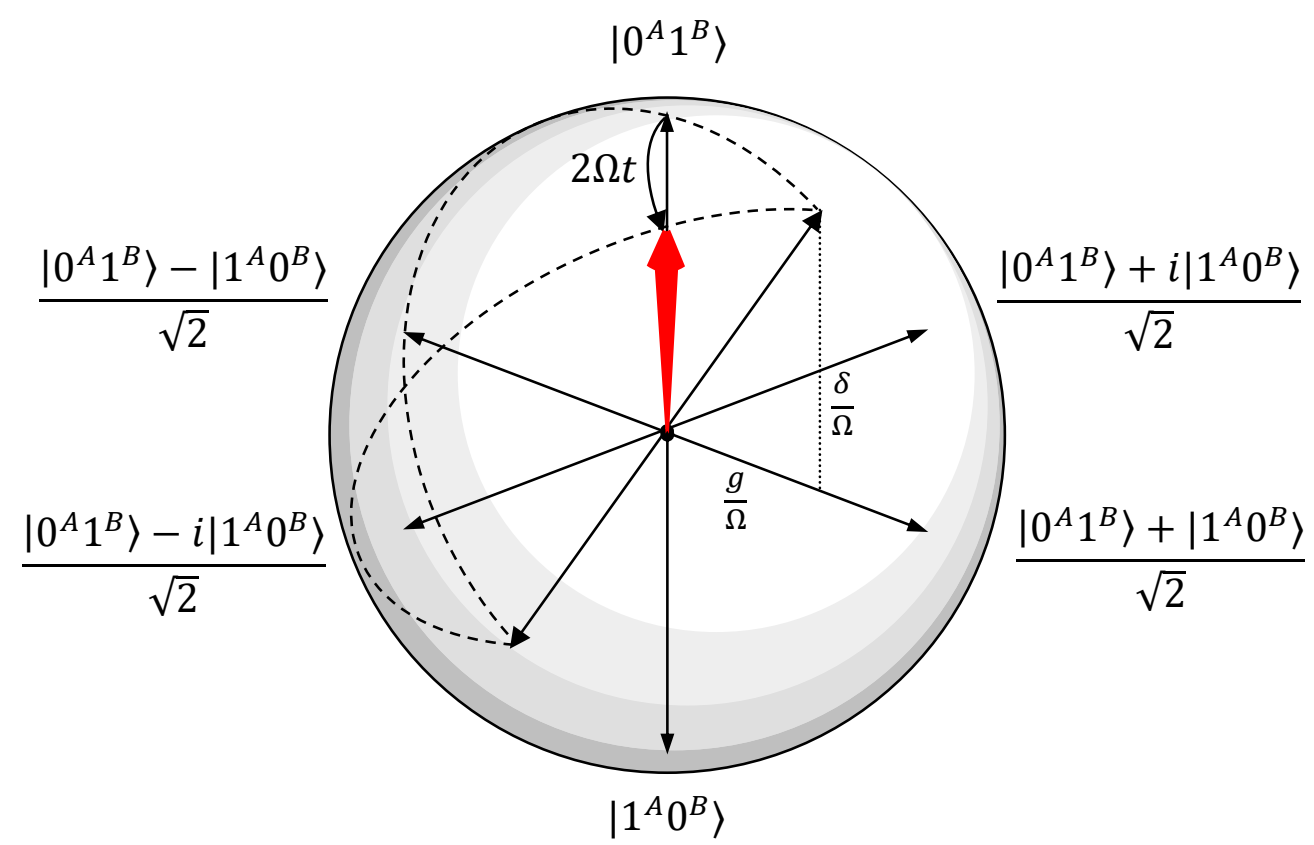

107

108

109

110

111

112

113

114

115

116

117

118

119

120

121

122

123

124

125

126

127

128

129

130

Figure 6. Evolution of the $\left|0^{A} 1^{B}\right\rangle,\left|1^{A} 0^{B}\right\rangle$ manifold of states of an isolated 2-neuron system on the Bloch sphere. Compared with Figure 4, where the nervous energy of the one-action potential state is the same in neurons $A$ and $B$, here $\delta=\frac{\omega^{B}-\omega^{A}}{2}$ may be non-zero. As before $g$ parameterises the strength of synaptic coupling. The angular frequency of oscillatory flow of action potential between neurons $\Omega=\sqrt{g^{2}+\delta^{2}}$. Compared with the evolution of an initial 1 -action potential state of $B$ plotted in Figure $5 b$, here the nadir of spike potential occurs earlier and is less profound.

These dynamics describe the interaction between a single source and target neuron.

Only when we discard the fanciful model of two isolated but interacting neurons, and impose instead the constraints of realistic neuronal connectivity, do these dynamics yield the constant initial firing rate of the source that we require. The argument for a linear accumulation of spike probability in this latter case is based on a standard derivation [10] of 'Fermi's Golden Rule' [11] in the parallel quantum mechanical formalism, and will be presented here only in outline. In fact a single neuron is likely to provide synaptic input to a large number of target neurons. The prior probability that a single spike in the source will ultimately lead to a spike in any of one of these targets will be extremely small. We will assume a constant weak coupling $g$ between a single source neuron $B$ and any one of a large number of targets $A$.

If each target neuron were initially in the ground state then collectively they would act as a reservoir: if $t$ remains small, the probability of spike occurrence in $B$ could be approximated as the sum of weights for the $\left|1^{A}\right\rangle\left\langle 1^{A}\right|$ state in each $\rho^{A}(t)$. In a target neuron with similar angular velocity to the source, the weight of this state initially increases as 


$$
\frac{g^{2}}{g^{2}+\delta^{2}} \sin ^{2} \Omega t \cong g^{2} t^{2} \quad\left(\delta^{2} \cong 0, t \text { small }\right)
$$

132 as in Figure 5b. Meanwhile, in a target neuron with angular velocity $\omega^{A}$ very different 133 to $\omega^{B}$, the weight of the $\left|1^{A}\right\rangle\left\langle 1^{A}\right|$ state becomes a 'sinc-squared' function of $\delta$ :

$$
\frac{g^{2}}{g^{2}+\delta^{2}} \sin ^{2} \Omega t \cong g^{2} \frac{\sin ^{2} \delta t}{\delta^{2}} \quad\left(g^{2} \ll \delta^{2}\right)
$$

136 that there are so many target neurons that the distribution of target angular velocities $\omega^{A}$

137 around $\omega^{B}$ can be considered continuous, and moreover that the number of target

138 neurons within a narrow range $\Delta \omega^{A}$ is relatively constant at $q$, the combination of these

139 effects that leads to a linear accumulation of spike probability:

$$
P(t)=\pi q g^{2} t
$$

141 The initial firing rate of the 1-action potential state in $B$ is constant, as we require (Figure $1425 \mathrm{a})$

3.8. Temporal frequency of the stream of consciousness

$w$ is the rate constant of an exponential decay in the spike potential of the source, expressed in terms of the strength and density of synapses on target neurons. Experience will also have 'relaxation' dynamics, in which a proportion $\frac{1}{e}$ of the initial perception

148 value decays within an average spike time of $\frac{1}{w}$. In effect, $w$ is the maximal temporal

149 frequency of the stream of consciousness. $\frac{1}{w}$ is also the minimal temporal resolution of 150 spike trace recording that is sufficient to make reliable inferences about consciousness. 151 For example, an estimate of spike frequency (across a finite number of trials) in bins of width less than $\frac{1}{w}$ is sufficient to predict the continuously evolving perception value with reasonable confidence. If the instantaneous firing rates of two neurons $A$ and $B$ are 154 dependent, so that

$$
\frac{f_{A B}(t)}{f_{A}(t) f_{B}(t)} \neq 1
$$

156 then some dependence in firing is likely to persist for at least a period $\frac{1}{w}$. This can be 157 thought of as the fundamental temporal (im)precision of firing correlation. A metric of 158 paired association between spike counts in coincident bins of width $\frac{1}{w}$ will capture the 159 qualitative proximity between neural elements of experience [3] 
163

164

165

166

\section{7}

168

169

170

171

172

173

174

175

176

177

178

179

180

181

182

183

184

185

186

187

188

189

190

191

relation of quantum mechanics) the perception value of the source neuron is singlevalued at an instant in time. Moreover, provided that we consider a closed neural system that includes the source and all target neurons, the evolution of the mental state will still be unitary and completely deterministic.

\section{Discussion}

While this paper has focused narrowly on neural state evolution, it is the third of a triptych of papers outlining the author's new model of consciousness (see references [1] and [3]). At this point it is worth taking stock of what that model has achieved, and defending some of the decisions that have been made in its development. After a brief summary of the model, the first part of this Discussion will reflect again upon how the model characterises the subjective representation of information, and its relationship to the neural code. The second part reconsiders the quantum aspects of the model, and implications for the function of consciousness; the final part the primitive evolution of the isolated neuron characterised by $\omega$, and neural system evolution generated by synaptic interaction. The Conclusions anticipate future directions of model development.

\subsection{Model summary}

Three features of consciousness are considered to be fundamental: subjectivity, unity and smooth evolution. A model of a consciousness with these features is constrained by three bridging principles, this term and the modelling approach originally suggested by Chalmers [12]. All three principles posit the subjective mirroring of objective neural information processing: the first relates single neuronal activity to a neural element of experience, the second relates paired association in neural activity to the qualitative relationship between such neural elements, the third relates the dynamics of both consciousness and objective neural representation to synaptic transmission. The instantaneous firing rate and firing rate dependence are taken to be the relevant measures of single neuronal activity and paired association between neurons respectively. The model uses a quantum mechanical mathematical formalism, adapted to the inter-neuronal synaptic level of interaction, to reconcile the existence of a mental state, having the three fundamental features, with these relevant objective measures of neural activity.

\subsection{Subjective representation and the neural code}

The definition of consciousness adopted in this paper is a general one provided by Searle [13]: "Consciousness consists of inner, qualitative, subjective states and processes of sentience or awareness." It is worth noting that this descriptive domain, the scope of the current model, is much broader than that considered by previous pioneers of quantum consciousness, whose ideas on conscious evolution will be discussed below. Penrose [14] concentrates more narrowly on conceptual rather than perceptual experience, particularly the dawning of awareness of a solution to a difficult problem, which seems to arise abruptly from unconscious processing. Stapp, on the other hand, refers to both a stable volitional state of consciousness, and reflections upon primitive 
experiences [15]. It is the author's expectation that Searle's broader consciousness, when analysed through introspection by other human subjects, will be found to have the same three features that the author considers fundamental: it seems likely, given the common neuro-anatomy and physiology between humans, that the essential qualities of experience are shared. On the other hand, it is possible that the three fundamentals reflect the author's particular cultural influences. Nevertheless, their statement seems important, to establish terms of reference for the scheme.

Even if it were common to most subjects, one of these features of consciousness might be claimed to be illusory, and the foundations of the model claimed unsound as a result. For example a critic might assert that "The continuous evolution of my experience is an illusion". Such criticism is easier to defend. If we define an illusion to be a subjective experience that deviates from reality, then the statement is as internally inconsistent as the assertion "This statement is false". If the reality is that my experience is discontinuous, then there is no such thing as "the continuous evolution of my experience". Conversely, if I do indeed experience continuous evolution, then this is a subjective reality, and there is no illusion.

One might also question the validity of one or all of the three bridging principles. In his original exposition, Chalmers considered "bridging principles . . . basic elements to our theory, not to be further explained" that "add the minimal component" to objective neuroscience to "bring subjective experience in" [12]. In the case of the three bridging principles above, contemplation of a model of consciousness not bound by neural constraints will be the only 'further explanation', offered not so much as proof by contradiction, but as a parsimony defence. That chemical synaptic transmission is a cardinal form of communication between neurons is beyond serious neuroscientific dispute. There is also a weight of neuroscientific evidence that neurons represent information in their firing rates and firing dependencies. Within this body of evidence, there is an extensive literature that supports these representations being neural correlates of consciousness in a range of experimental paradigms. So if consciousness is not neural, then there must be two parallel pathways, one objective and one subjective, both representing and transmitting the same information, separate but correlated. If this does not already seem extravagant, consider that the information processing in the subjective pathway would still need to influence behavioural outputs expressed as spike patterns in motor cortex, lest consciousness were to be relegated to the status of epiphenomenon.

\subsection{Quantum aspects and implications}

The major achievement of the current model is the reconciliation of a mathematical formalism, suited to the description of consciousness, with the classical neuron doctrine of spikes and synaptic transmission. The model's most serious potential pitfall is that quantum mechanics, from which the formalism is borrowed, usually applies at a vastly smaller physical scale. This is the level of certain biologic particles, fields and their 
interactions, in the quantum physical description of which, others have attempted to include consciousness. Here the fundamental units are not spikes but the atomic-level particles of a quantum many-particle system, for example the unbound electrons (fermions) of Valentine [16] or the Nambu-Goldstone boson modes of Vitiello [17]. Although the mathematical formalism of quantum field theory underlying such models is well developed, the connection between the condensation of quantum fields and neuronal membrane depolarisation often remains obscure, or at least nebulous: "a wave packet ... acting as a bridge from quantum dynamics at the atomic level through the microscopic pulse trains of neurons to the macroscopic properties of large populations of neurons" [18].

There is one other reason why quantum mechanics has been attractive for theorists of consciousness: it has the potential to exploit a loophole in the causal closure contract of classical neuroscience, thereby allowing consciousness a functional role. Chalmers argues that to make the assumption that there are functional properties that distinguish a brain endowed with conscious experience is to ignore, or at least trivialise the 'hard problem' [19]. According to this view, it would be impossible both to describe consciousness adequately and to ascribe some function to it. It is true that if a model were to capture the private and subjective quality of the conscious state, then the representation of that state could not also be a directly observable neural property. However, it is not generally the case that states or realities that are not directly observable have no statistically measurable objective effects. The quantum mathematical formalism of the current model expresses this duality even at a single neuron level. The perception value is a subjective reality that exists when a sensory neuron responds to a stimulus, but is inaccessible directly to neurophysiologic observation (see section 1.2). The instantaneous firing rate, over an ensemble of stereotyped stimulus presentations, is the statistical objective effect.

So what then is this function of consciousness? We will consider this first from a systems-neuroscience and second from a single cell perspective. It is common in neuroscientific theorising to hear perceptual experience described as distillation of order from the chaos of sensory input. Koch speculates that "Natural selection pursued a strategy that amounts to summarizing most of the pertinent facts about the outside world compactly and sending this description to the planning stages to consider the organism's optimal course of action" ([20], 14.1). In the current model, this summary of pertinent facts could be exponentially more compact than a purely classical neuronal register. A linear increase in the number of neurons would be associated with an exponential increase in the dimension of the tensor product of neural spaces. Just as a single neuron could be in a superposition of 0 - and 1-action potential states, a pure state of $m$ neurons could be in a superposition of at least $2^{m}$ states of definite spike potential. Perhaps then, consideration of 'optimal course of action' is quantum computation on all this data. Conceivably, myriad synaptic interactions that evolve a pure mental state may drive an exponential number of parallel computations upon the sensory input, which might ultimately interfere as some desirable behavioural result. 
The model should also allow correlations between neurons in anatomically remote higher-order sensory cortices that have no direct synaptic connection with each other, correlations beyond what could have been established classically by common synaptic input from some lower level of the sensory hierarchy. One would anticipate an increased efficiency of spike generation afforded by synchronous inputs to a common target neuron in motor cortex: "100 fast excitatory inputs, distributed over the dendritic tree of a large pyramidal neuron, are sufficient to generate ...(a spike)...if they are activated within a millisecond of each other. If the presynaptic spikes arrive smeared out over a $25 \mathrm{msec}$ window, however, twice as many synapses are needed to fire the cell" ([20], 2.3).

A caveat to this discussion is that these putative benefits to brain function are nonlocal effects of a pure mental state. They depend on the quantum mathematical formalism applying simultaneously to interactions ongoing at two entirely different phy sical scales, the atomic scale of quantum physics and the microscopic scale of synaptic transmission (see Conclusions). Popescu's application of a quantum model to statistical mechanics sets a precedent for this kind of approach, offering an ontic rather than epistemic interpretation of the probabilities that define entropy [21]. Applied to the context of neuroscience, these results lend credence to the idea that we might just as well derive firing probabilities from a density operator that is the reduction of a definite pure mental state as regard these probabilities as a manifestation of our lack of knowledge about of the actual state of the brain.

\subsection{Evolution of a closed neural system}

If a model is to allow the state of a 'closed' multi-neuron system, lacking synaptic interaction with external neurons, continuously to evolve (see Conclusions), then it must also allow the individual neuronal constituents of that system, when isolated, to evolve continuously in a more limited way. This was the origin of the idea of an immutable but neuron-specific $\omega$, a rate of acquisition of relative phase of a 1-action potential state of the neuron. A critical step in the model's further development was the introduction of nervous energy, bringing together seemingly disparate concepts of a neuron's contribution to consciousness and its dynamics: the operator, the expectation value of which describes the magnitude of a neural element of experience, is the very same one that generates isolated neuronal evolution. In order for the perception value and instantaneous firing rate of a source neuron to remain proportional, in the process of synaptic transmission modelled as action potential flow, a reservoir of target neurons of differing $\omega$ was found to be necessary. These target neurons then must also differ according to $\omega$ in their contribution to consciousness, heuristically in magnitude of experience-per-spike.

For the current computational model of brain to be implemented in the future, a potential objective neural property constraining $\omega$ will need to be explored. The conjecture of the author is that this may be related in some way to a neuron's objective 
size. When the vibrating length of an open violin A-string is shortened or 'stopped' by a finger, its fundamental frequency or pitch rises, perhaps to B. Considering the A and B fundamentals to be analogous to the 1-action potential $\omega$ of a larger or smaller neuron respectively, natural/artificial harmonics created by lesser finger pressure at $\frac{1}{2}, \frac{1}{3}, \frac{1}{4}$ of the length of the string could be considered analogues of 2-, 3-, 4-action potential states.

\subsection{Evolution by synaptic interaction: measurement and decoherence}

Nervous energy transfer in chemical synaptic transmission, as proposed, is analogous to radiative energy dissipation by photon emission. Indeed, the model of synaptic interaction between one source and many target neurons is formally identical to the interaction of an atom in its first exited state with an environment, consisting of a reservoir of simple harmonic oscillators prepared in the ground state. Experimental interrogation of the environment for the occurrence of photon emission could be viewed as causing a collapse of the atom to one or other definite energy state, which would not have occurred without the experimenter's intervention. However, if the interaction between atom and environment admits an interpretation as a unitary evolution on the combined system, there must simultaneously be photon emissions as discrete events, even as the energy of the atom decays continuously. Analogously, in the current model of neural dynamics, we allow spikes as discrete events, even as the nervous energy decays continuously. We have adopted here not only a quantum mathematical formalism, but a decoherence theoretic interpretation of quantum mechanics that sees measurement as a local manifestation of unitary interaction between system and environment [7]. Although a quantum system undergoes an abrupt objective transition (with a probability that is usually regarded as irreducible), the evolution of a pure state of system and environment remains strictly deterministic.

When this model is extended to the level of the complex neural system that is the whole brain, a multitude of synaptic interactions between component neurons generates a continuous unitary evolution of a coherent mental state in a tensor product neural space, which is associated with the 'stream of consciousness'. This clearly conflicts with other quantum theories $[14,15]$, which attribute 'moments' of consciousness to collapse of the state vector. This difference reflects both divergent concepts of consciousness and interpretations of quantum mechanics.

Stapp stays true to von Neumann's original division of quantum evolution into three processes [22]. Applied to brain dynamics, Process 2 allows a superposition of physical brain states to undergo unitary evolution until Process 3 intervenes such that the superposition collapses to one specific physical state. In sensory perception, Stapp imagines that a simple task of a sensory system may be to answer the question "is a particular stimulus present?" If the system collapses to a neural representation of that stimulus, the organism experiences "the conscious acquisition of the knowledge associated with that answer 'Yes.'”[23]. Process 1 is, according to von Neumann [22], the 
determination of which question to ask, presumably in the case of brain dynamics a direct consequence of the definite physical state of the brain that was realised by the last Process 3 event.

Penrose [14] characterises von Neumann's Process 3 as reduction $\mathbf{R}$ of a superposition state that has previously evolved deterministically according to unitary evolution $\mathbf{U}$ (Process 2). The issue of when $\mathbf{R}$ supervenes upon $\mathbf{U}$ is highlighted as the measurement problem. Penrose speculates that $\mathbf{R}$ occurs whenever the states in superposition diverge sufficiently in space-time to meet an objective threshold, hence objective reduction OR. In the brain, Hameroff and Penrose [24] suggest that, via U interactions between adjacent subunits, microtubule superpositions extend across significant regions of the brain, before OR occurs as a moment of consciousness. Stapp [23], eschewing an objective criterion, allows 'conscious effort' an influence in limiting the duration of unitary evolution (Process 2), thereby holding the focus of attention before collapse occurs (Process 3 ). The experience of effort is, according to this suggestion, something different from the experience of everything else (Process 3 ).

For other theorists, it is the experience of freely willed action that seems abrupt and random, and that is modelled as a non-deterministic measurement process. Like Stapp, Beck and Eccles [25] would like to give consciousness agency in influencing neural events. To do so they propose a "mechanism (that) clearly lies beyond ordinary quantum mechanics" [25]. For these authors, quantum measurement takes place at the synapse, particularly in the supplementary motor cortex involved in planning movement. In contrast to Stapp, their metaphysical force influences the probability of synaptic vesicle release, a probability that would be irreducible in the standard quantum mechanical sense: "the mental intention (the volition) becomes neurally effective by momentarily increasing the probability of exocytosis in selected cortical areas" [25].

There has been telling criticism of previous quantum models of neural signalling. Tegmark [26] equates objective states of neuronal polarisation or depolarisation to the decoherence of about $10^{6}$ sodium ions into a definite position outside or inside the membrane respectively. He calculates that decoherence occurs within a time (T2) of order $10^{-19}$ seconds, much less than the "characteristic dynamical time scale of cognitive processes", which he estimates to be somewhere between $10^{-2}$ and $10^{0}$ seconds. The implications are that pure superposition states do not survive long enough even in a single neuron to allow quantum computation, and certainly not across the "complex network of $\sim 10^{11}$ neurons ...linked to our subjective perceptions" that we would need to invoke some unity of consciousness.

Even so, Tegmark argues that if one adopts, as we have done, the reasonable assumption that "different neuron firing patterns correspond in some way to different conscious perceptions, then consciousness itself cannot be of a quantum nature even if 
411 there is a yet undiscovered physical process in the brain with a very long decoherence

412 time...The reason is that as soon as such a quantum subsystem communicates with the 413 constantly decohering neurons to create conscious experience, everything decoheres"

414 [26]. This seems a powerful argument against any of the pre-existing quantum theories of 415 consciousness mentioned above.

\section{5. Conclusions}

417 The formalism of consciousness developed in this paper preserves a pure mental 418 state because it is limited to a description of interactions between neurons. We have 419 chosen to treat the brain as an isolated system precisely so that we can maintain the 420 picture of the unity of consciousness. The flow of action potential in synaptic

421 transmission is equivalent to nervous energy dissipation, rendering reduced states of 422 single neurons mixed [3], and quantum effects negligible at the single cell level.

423 Superpositions of 0 - and 1-action potential states, of neural histories in which a spike has 424 and has not occurred, are not evident to the neuroscientist. The lack of any local neuronal 425 footprint of consciousness is also entirely consistent with the theory. However, prediction 426 of quantum effects at a neural system or whole brain level will depend on how the 427 formalism can be reconciled with quantum physics: specifically, how quantum coherence 428 might be preserved within a neural system, in the face of continuous interaction between neurons and their extracellular environment. Perhaps it is evolution that has resolved this paradox, through natural selection of a concatenated neural architecture to refocus the mental state on a conscious neuronal subspace, thereby achieving a superlative feat of quantum error correction. Only if this turns out to be possible could one start to consider attributing experience to a non-biologic quantum computer. However, even if such attempts at reconciliation ultimately prove futile, it is hoped that they will illuminate the way in which standard quantum mechanics may require modification to become a satisfactory description of consciousness.

Quite apart from these big questions of context of the current theory in contemporary science, there remains, of course, much to be done to extend the model to ever more realistic neural systems. Firstly we need to acknowledge that spikes rarely occur as isolated events. Future work will need to relate the decay of superposition states of multiple action potentials to the probability distribution of multiple-spike trains.

Secondly, there is a need to explore how a directional flow of action potential through a neuron can be modelled. All that has been described in the current paper is the continuous evolution of a neural element of experience that parallels objective discontinuous spike formation and synaptic transmission. Considering the subjective aspect, the specific starting condition considered in the paper, one action potential in the source neuron and no action potentials in a reservoir of target neurons, is a highly structured state. In an information theoretic sense, the entropy of the combined subjective state increases through synaptic transmission. The dynamics that ensue from this specific starting condition are 
indeed irreversible in time. It is acknowledged, however, that there is more to the story that will need to be told in future work. The limitation of the current model that it seemed to allow bidirectional or reversible flow of action potential through the neural circuit: not only synaptic transmission forward from axon to dendrite, but backward from dendrite to axon. Moreover, in what has been presented, there is a lack of sustainability of evolution in time. In time, all neurons connected by synapses would relax to about the same spike potential: a maximum entropy state of brain.

Examining the sub-cellular objective dynamics that correlate with the evolution of experience that has been modelled (see section 1.2), they are found to be largely passive (not energy-requiring), involving the discharge of transmembrane potential. In contrast, the other component of objective dynamics that is yet to be modelled, membrane repolarisation (also neurotransmitter synthesis or reuptake) is ATP-dependent. The energy debt incurred is ultimately repaid through cellular metabolism. The processes of glycolysis and oxidative phosphorylation that transform ADP back to ATP also generate heat. In a thermodynamic sense, it is these other aspects of subcellular evolution that are irreversible.

These considerations motivate the following sketch of a future extended model of neural states and their evolution. I suspect that to describe unidirectional action potential flow and a sustained stream of consciousness, a model with at minimum two neural compartments would be required. We could label these compartments 'dendritic' and 'axonal' in order to indicate that synaptic transmission is to be represented by action potential flow from the axonal compartment of the source to the dendritic compartment of the target neuron, but these compartments should not be associated too literally with objective functionally specialised parts of the neuron. The extended 2-compartment model would also include a new continuous process that would pump or shunt action potential unidirectionally from dendritic to axonal compartments. One would hypothesise that without the effect of this pump, action potential flowing into the dendritic compartment by synaptic transmission from some other source would quickly become distributed between compartments. The operation of the pump would ensure that virtually none remained in the dendritic compartment, and would erase a bit of information about action potential location. By Landauer's Principle, such information erasure would be dissipative [27]. The objective neural correlate would be the energy expenditure per spike of membrane repolarisation, mentioned above. Note that the model of consciousness as described above would otherwise remain unaltered: the perception value of the neuron would still be the expectation value of the number of action potentials; the stream of consciousness would still be generated by synaptic interaction.

Finally, we will consider here, at least in broad conceptual terms, the evolution of a complex neural system such as the brain, which is not closed even in the limited sense of synaptic interaction, but which communicates with the outside world via a peripheral nervous system. Even if we assume that physical interactions within the central nervous 
system are unchanging, the nervous energy that generates the evolution of the mental state still cannot be time-independent. As we extend our definition of brain to include structures that seem ever less likely to support consciousness, such as thalamic relay nuclei, spinal cord and dorsal root ganglia, external influences will still modulate the potential of component neurons to fire. According to the model we have developed, there is still a flow of action potential to and from the composite neural system.

In our description of source and target neurons detailed above, we could have predicted the same evolution of spike potential if we had modelled synaptic interaction as a small oscillatory perturbation of the nervous energy of the source at angular frequency $\Omega$ [28]. In this approximation, the state of the source neuron would then remain pure, undergoing unitary evolution, but with a time-dependent nervous energy. By analogy, we propose that taking any reasonable definition of brain, the neural interaction with the periphery, modelled as a perturbation of the composite nervous energy, is sufficiently small and slowly changing that unitary evolution of a mental state vector remains a reasonable approximation of the conscious state of the brain. If the perturbation of the nervous energy represents the interaction of brain with the sensory environment, generating 'perceptual' experience, then perhaps the unitary evolution generated by time-independent interactions within brain is the 'conceptual' component of the stream of consciousness.

Supplementary Materials: There is no supplementary material available online.

Funding: This research received no external funding.

Conflicts of Interest: The author declares no conflict of interest.

\section{References}

1. Duggins, A.J. A complex vector space model of single neuronal coding and experience. BioSystems 2010, 102, 124-133.

2. Britten, K.H.; Shadlen, M.N.; Newsome, W.T.; Movshon, J.A. The analysis of visual motion: a comparison of neuronal and psychophysical performance. J. Neurosci. 1992, 12, 4745-4765.

3. Duggins, A.J. Pure state consciousness and its local reduction to neuronal space. AIP Conference Proceedings 2013, 1510, 162-181.

4. Zeki, S.; Bartels, A. Toward a theory of visual consciousness. Conscious Cogn. 1999, 8, 225-59.

5. Schrödinger, E. Mind and matter. Cambridge University Press: Cambridge, UK, 1958.

6. Nagel, T. What is the mind body problem? In Experimental and theoretical studies of consciousness; Ciba Foundation symposium. Vol 174, Wiley: New York, USA, 1993.

7. Zurek, W.H. Decoherence and the transition from quantum to classical. Physics Today 1991, 44, 36-44.

8. Jaynes, E.T.; Cummings, F.W. Comparison of quantum and semiclassical radiation theories with application to the beam maser. Proc. IEEE 1963, 51, 89-109.

9. Lecture Notes for Physics 229: Foundations I: States and Ensembles; John Preskill. Available online: http://www.theory.caltech.edu/people/preskill/ph229/\#lecture (accessed on 27 December 2019).

10. Nielsen, M.; Chuang, I. Quantum computation and quantum information, 1st ed.; Cambridge University Press: Cambridge, UK, 2000.

11. Dirac, P.A. The Quantum Theory of Emission and Absorption of Radiation. Proceedings of the Royal Society A 1927, 114, 243-265.

12. Chalmers, D.J. The problems of consciousness. Adv neurol 1998, 77, 7-16. 
539 13. Searle, J.R. Consciousness. Annual Review of Neuroscience 2000, 23, 557-578.

540 14. Penrose, R. Shadows of the mind: a search for the missing science of consciousness, 1st ed.; Random $541 \quad$ House: London, UK, 1995.

542 15. Stapp, H.P. Mind, Matter and Quantum Mechanics. Foundations of Physics 1982, 12, 363-399.

543 16. Valentine, J.D. Towards a physics of consciousness. Psychoenergetics 1982, 4, 257.

544 17. Vitiello, G. Quantum dissipation and information: A route to consciousness modeling. $545 \quad$ NeuroQuantology 2003, 2, 266-279.

546 18. Freeman, W.J.; Vitiello, G. Nonlinear brain dynamics as macroscopic manifestation of underlying many-body field dynamics. Physics of Life Reviews 2006, 3, 93-118.

19. Chalmers, D. The conscious mind: in search of a fundamental theory; Oxford University Press: New York, USA, 1996.

20. Koch, C. The quest for consciousness: a neurobiological approach; Roberts: CO, 2004.

21. Popescu, S.; Short, A.J.; Winter, A. Entanglement and the foundations of statistical mechanics. Nature Physics 2006, 2, 754-758.

22. von Neumann, J. Mathematical Foundations of Quantum Mechanics, translated by Beyer RT; Princeton University Press: Princeton, USA, 1955.

23. Stapp, H. Quantum Approaches to Consciousness. In Cambridge Handbook of Consciousness; Moscovitch, M., Zelazo, P., Thompson, E., Eds.; Cambridge University Press: New York, USA, 2007.

24. Hameroff, S.; Penrose, R. Conscious events as orchestrated space-time selections. Journal of Consciousness Studies 1996, 3, 36-53.

25. Beck, F.; Eccles, J.C. Quantum aspects of brain activity and the role of consciousness. Proc Natl Acad Sci USA 1992, 89, 11357-61.

26. Tegmark, M. Importance of quantum decoherence in brain processes. Phys Rev E Stat Phys Plasmas Fluids Relat Interdiscip Topics 2000, 61, 4194-206.

27. Landauer, R. Irreversibility and heat generation in the computing process. IBM J Res Dev 1961, $5,183$.

28. Davies, P.; Betts, D. Quantum mechanics; Stanley Thornes: Cheltenham, UK, 1999. 